\title{
Design of fuzzy power system stabilisers for multimachine power systems
}

Y.-Y. Hsu, BSc, MSc, PhD

C.-H. Cheng, BSc, MSc

\begin{abstract}
A new type of power system stabiliser based on fuzzy set theory is proposed to improve the dynamic performance of a multimachine power system. To have good damping characteristic over a wide range of operating conditions, speed deviation $(\Delta \omega)$ and acceleration $(\Delta \dot{\omega})$ of a machine are chosen as the input signals to the fuzzy stabiliser on that particular machine. These input signals are first characterised by a set of linguistic variables using fuzzy set notations. The fuzzy relation matrix, which gives the relationship between stabiliser inputs and stabiliser output, allows a set of fuzzy logic operations that are performed on stabiliser inputs to obtain the desired stabiliser output. Since only local measurements are required by the fuzzy stabiliser on each generating unit, the proposed stabiliser is of decentralised output feedback form and is easy for practical implementation. The effectiveness of the proposed fuzzy stabiliser is demonstrated by a multimachine system example.
\end{abstract}

\section{Introduction}

The application of power system stabilisers (PSS) to improve the dynamic performance of a power system has been the focus of extensive studies for more than two decades [1-8]. The most widely employed power system stabiliser is the lead-lag PSS where the gain settings are fixed at certain values which are determined under particular operating conditions. Since the operating point of a power system drifts as a result of continuous load changes or unpredictable major disturbances such as a three-phase fault, it is desirable to adapt the stabiliser parameters in real-time based on on-line measurements in order to maintain good dynamic performance over a wide range of operating conditions. This motivates the development of a self-tuning power system stabiliser [9-14].

Although the self-tuning PSS is capable of offering better dynamic performance than a fixed-gain PSS, it suffers from a major drawback of requiring model identification in real-time which is very time consuming, especially for a microcomputer with limited computational capability.

Paper 7206C (P4, P9), first received 14th August and in revised form 19th December 1989

The authors are with the Department of Electrical Engineering, National Taiwan University, Taipei, Taiwan

IEE PROCEEDINGS, Vol. 137, Pt. C, No. 3, MAY 1990
In this paper, a new type of power system stabiliser, which does not require real-time model identification, is developed using fuzzy set theory [15]. The proposed fuzzy PSS uses two real-time measurements $\Delta \omega$ (generator speed deviation) and $\Delta \dot{\omega}$ (acceleration) as the input signals. To determine stabiliser output, these input signals are first described by some linguistic variables such as $L P$ (large positive), $M P$ (medium positive), $S P$ (small positive), $V S$ (very small), $S N$ (small negative), $M N$ (medium negative), and $L N$ (large negative), using fuzzy set notation. A fuzzy relation matrix which gives the relationship between stabiliser inputs and stabiliser output is then developed using membership functions. A set of fuzzy logic operations are performed to yield the desired stabiliser output based on this fuzzy relation matrix. The effectiveness of the proposed fuzzy PSS is demonstrated by examining the dynamic performance of a multimachine power system subject to a major disturbance.

The main features of the work are summarised as follows.

(a) The developed fuzzy PSS is very efficient and can be easily implimented on a microcomputer since it does not require real-time model identification.

(b) The fuzzy stabiliser developed in this paper is of decentralised output feedback form since only local measurements $(\Delta \omega$ and $\Delta \dot{\omega})$ are employed as the inputs to each PSS.

(c) Results from the application of the fuzzy PSS to a multimachine system reveal that the fuzzy PSS can offer good dynamic performance over a wide range of operating conditions.

\section{Introduction to fuzzy set theory}

Some basic definitions and mathematical operations of fuzzy sets are first described [15].

\subsection{Fuzzy set}

Let $X$ be a collection of objects ( $X$ is the universal set), then a fuzzy set $A$ in $X$ is defined to be a set of ordered pairs

$$
A=\left\{\left(x, \mu_{A}(x)\right) \mid x \in X\right\}
$$

where $\mu_{A}(x)$ is called the membership function of $x$ in $A$.

Note that the membership function $\mu_{A}(x)$ denotes the degree to which $x$ belongs to $\tilde{A}$ and is normally limited to values between 0 and 1 . A high value of $\mu_{\bar{A}}(x)$ implies that it is very likely for $x$ to be in $A$. Elements with a zero degree of membership are normally not listed. If we limit the values of the membership function to be either 0 or 1 , then $A$ becomes a crisp (nonfuzzy) set. 


\subsection{The AND operator (The intersection of two fuzzy} sets)

Let $A$ and $B$ be two fuzzy sets with membership functions $\mu_{A}(x)$ and $\mu_{\mathfrak{B}}(x)$, respectively. The membership function of the intersection (AND), $C=A \cap B$, is defined by

$$
\mu_{C}(x)=\min \left(\mu_{A}(x) \quad \mu_{B}(x)\right) \quad x \in X
$$

2.3 The OR operator (The union of two fuzzy sets) Let $A$ and $B$ be two fuzzy sets with membership functions $\mu_{A}(x)$ and $\mu_{B}(x)$, respectively. The membership function of the union (OR), $D=A \cup B$, is defined by

$$
\mu_{D}(x)=\max \left(\mu_{A}(x) \quad \mu_{B}(x)\right) \quad x \in X
$$

\subsection{The NOT operator (The complement of a fuzzy} set)

Let $A$ be a fuzzy set with membership function $\mu_{A}(x)$. The membership function of the complement of $A$, is defined by

$$
\mu_{C A}(x)=1-\mu_{A}(x) \quad x \in X
$$

\subsection{Fuzzy relation}

Let $A$ and $B$ be two fuzzy sets with membership functions $\mu_{A}(x)$ and $\mu_{B}(x)$, respectively. A fuzzy relation $R$ from $A$ to $B$ can be visualised as a fuzzy graph and can be characterised by the membership function $\mu_{R}(x, y)$ which satisfies the composition rule as follows:

$$
\mu_{B}(y)=\max _{x}\left(\min \left(\mu_{R}(x, y) \quad \mu_{A}(x)\right)\right)
$$

\section{Design of the fuzzy power system stabiliser}

To make the designed PSS capable of providing the desired system damping under disturbance conditions, some state variables representative of system dynamic performance must be taken as the input signals to the fuzzy PSS. In this work, generator speed deviation $(\Delta \omega)$ and acceleration $(\Delta \dot{\omega})$ are chosen to be input signals of the fuzzy PSS since our previous experience indicated that, under disturbance conditions, dynamic performance of the system could be evaluated by examining the response curves of these two variables.

In practice, only shaft speed $(\Delta \omega)$ is readily available [16]. The acceleration signal $(\Delta \dot{\omega})$ can be derived from the speed signals measured at two successive sampling instants using the following equation $[17,18]$ :

$$
\Delta \dot{\omega}(k)=(\Delta \omega(k)-\Delta \omega(k-1)) / \Delta T
$$

where $\Delta T$ is the sampling interval. To limit the impact of noise and minimise torsional interaction, low pass or band-reject filters are required in practical implementation [1].

To determine the stabiliser output from the measured system variables $\Delta \omega$ and $\Delta \dot{\omega}$, a fuzzy relation matrix $\boldsymbol{R}$, which gives the relationship between the fuzzy set characterising stabiliser inputs and the fuzzy set characterising stabiliser output, is first established and stored in computer memory. The fuzzy PSS proceeds as follows to evaluate the desired output signal.

Step 1: Use membership functions to represent stabiliser inputs $\Delta \omega$ and $\Delta \dot{\omega}$ in fuzzy set notation.

Step 2: Use the composition rule in eqn. 5 to determine the membership function of the stabiliser output $V_{\text {PSs }}$.

Step 3: Determine a proper stabiliser output from the membership function of the output signal.
Details of the above procedures are addressed in the following discussions.

\subsection{Establish the fuzzy relation matrix}

A fuzzy relation matrix must be set up and stored in computer memory. A set of decision rules relating stabiliser inputs to stabiliser output are first compiled based on our previous experience with stabiliser design [5-8, 11-13]. These decision rules are expressed using linguistic variables such as large positive $(L P)$, medium positive $(M P)$, small positive $(S P)$, very small $(V S)$, small negative $(S N)$, medium negative $(M N)$, and large negative $(L N)$. For example, a typical rule reads as follows:

Rule 1 If $\Delta \omega$ is $L P$ and $\Delta \dot{\omega}$ is $L N$, then $V_{\mathrm{PSS}}$ (stabiliser output) should be $V S$.

Through the combination of the two input signals $\Delta \omega$ and $\Delta \dot{\omega}$, there will be 49 decision rules in all. The most convenient way to present these decision rules is to use a

\begin{tabular}{|c|c|c|c|c|c|c|c|}
\hline \multirow{2}{*}{$\begin{array}{l}\text { speed } \\
\text { deviation, } \Delta \omega\end{array}$} & \multicolumn{7}{|c|}{ Acceleration, $\Delta \dot{\omega}$} \\
\hline & $L N$ & $M N$ & SN & VS & $S P$ & $M P$ & $L P$ \\
\hline$L P$ & $V S^{*}$ & $S P$ & $M P$ & $L P$ & $L P$ & $L P$ & $L P$ \\
\hline$M P$ & $S N$ & vs & $S P$ & $M P$ & $M P$ & $L P$ & $L P$ \\
\hline$S P$ & MN & SN & VS & $S P$ & $S P$ & $M P$ & $L P$ \\
\hline VS & $M N$ & $M N$ & $S N$ & VS & $S P$ & $M P$ & $M P$ \\
\hline$S N$ & $L N$ & $M N$ & $S N$ & SN & vS & $S P$ & $M P$ \\
\hline$M N$ & $L N$ & $L N$ & $M N$ & $M N$ & $S N$ & vS & $S P$ \\
\hline$L N$ & $L N$ & $L N$ & $L N$ & $L N$ & $M N$ & $S N$ & vS \\
\hline
\end{tabular}
decision table as shown in Table 1. The decision table, which has been adopted from Reference 19, is found to be applicable to various problems involving decision making.

* Rule 1.

It is observed from Table 1 that each entry represents a particular rule. Using fuzzy set notations, the decision table in Table 1 can be converted into the fuzzy relation matrix in Table 2 where stabiliser output obtained by applying a particular rule is expressed in membership functions. For example, Rule 1 in eqn. 7 now becomes

Rule $1^{\prime}$ If $\Delta \omega$ is $L P$ and $\Delta \dot{\omega}$ is $L N$ then the stabiliser output $V_{\mathrm{PSS}}$ can be characterised by the fuzzy set $\{(L N, 0.0),(M N, 0.0)$, $(S N, 0.5),(V S, 1),(S P, 0.5),(M P, 0),(L P, 0)\}$.

Note that in Rule $1^{\prime}$ we are still not certain whether $\Delta \omega$ is $L P$ and $\Delta \dot{\omega}$ is $L N$. The degree of belief in the 'If' part ('condition part') of the rule can be specified through the use of membership functions as described below.

\subsection{Specify the membership functions for stabiliser inputs}

To express the stabiliser inputs in linguistic variables $L P$, $M P, S P, V S, S N, M N$, and $L N$, the measured stabiliser inputs $\Delta \omega$ and $\Delta \dot{\omega}$ are first normalised based on previous experience.

$$
\begin{aligned}
\Delta \omega_{u} & =\frac{\Delta \omega}{0.01} \\
\Delta \dot{\omega}_{u} & =\frac{\Delta \dot{\omega}}{0.001}
\end{aligned}
$$

Using these normalised quantities, stabiliser inputs can be described by membership functions for the linguistic

IEE PROCEEDINGS, Vol. 137, Pt. C, No. 3, MAY 1990 


\begin{tabular}{|c|c|c|c|c|c|c|c|c|}
\hline \multirow[t]{4}{*}{$x_{i}$} & \multirow{4}{*}{$\frac{\text { Stabiliser inputs }}{(\Delta \omega, \Delta \dot{\omega})}$} & \multicolumn{7}{|c|}{ Stabiliser output } \\
\hline & & $L N$ & $M N$ & $S N$ & $V S$ & $S P$ & $M P$ & $L P$ \\
\hline & & \multicolumn{7}{|c|}{ Membership value } \\
\hline & & $\mu_{R}\left(x_{i}, L N\right)$ & $\mu_{R}\left(x_{i}, M N\right)$ & $\mu_{A}\left(x_{i}, S N\right)$ & $\mu_{R}\left(x_{i}, V S\right)$ & $\mu_{R}\left(x_{i}, S P\right)$ & $\mu_{R}\left(x_{i}, M P\right)$ & $\mu_{R}\left(x_{i}, L P\right)$ \\
\hline$x_{1}$ & $(L P, L N)$ & 0 & 0 & 0.5 & 1 & 0.5 & 0 & 0 \\
\hline$x_{2}$ & $(L P, M N)$ & 0 & 0 & 0 & 0.5 & 1 & 0.5 & 0 \\
\hline$x_{3}$ & $(L P, S N)$ & 0 & 0 & 0 & 0 & 0.5 & 1 & 0.5 \\
\hline$x_{4}$ & $(L P, V S)$ & 0 & 0 & 0 & 0 & 0 & 0.5 & 1 \\
\hline$x_{5}$ & $(L P, S P)$ & 0 & 0 & 0 & 0 & 0 & 0.5 & 1 \\
\hline$x_{6}$ & $(L P, M P)$ & 0 & 0 & 0 & 0 & 0 & 0.5 & 1 \\
\hline$x_{7}$ & $(L P, L P)$ & 0 & 0 & 0 & 0 & 0 & 0.5 & 1 \\
\hline$x_{8}$ & $(M P, L N)$ & 0 & 0.5 & 1 & 0.5 & 0 & 0 & 0 \\
\hline$x_{9}$ & $(M P, M N)$ & 0 & 0 & 0.5 & 1 & 0.5 & 0 & 0 \\
\hline$x_{10}$ & $(M P, S N)$ & 0 & 0 & 0 & 0.5 & 1 & 0.5 & 0 \\
\hline$x_{11}$ & $(M P, V S)$ & 0 & 0 & 0 & 0 & 0.5 & 1 & 0.5 \\
\hline$x_{12}$ & $(M P, S P)$ & 0 & 0 & 0 & 0 & 0.5 & 1 & 0.5 \\
\hline$x_{13}$ & $(M P, M P)$ & 0 & 0 & 0 & 0 & 0 & 0.5 & 1 \\
\hline$x_{14}$ & $(M P, L P)$ & 0 & 0 & 0 & 0 & 0 & 0.5 & 1 \\
\hline$x_{15}$ & $(S P, L N)$ & 0.5 & 1 & 0.5 & 0 & 0 & 0 & 0 \\
\hline$x_{16}$ & $(S P, M N)$ & 0 & 0.5 & 1 & 0.5 & 0 & 0 & 0 \\
\hline$x_{17}$ & $(S P, S N)$ & 0 & 0 & 0.5 & 1 & 0.5 & 0 & 0 \\
\hline$x_{18}$ & $(S P, V S)$ & 0 & 0 & 0 & 0.5 & 1 & 0.5 & 0 \\
\hline$x_{19}$ & $(S P, S P)$ & 0 & 0 & 0 & 0.5 & 1 & 0.5 & 0 \\
\hline$x_{20}$ & $(S P, M P)$ & 0 & 0 & 0 & 0 & 0.5 & 1 & 0.5 \\
\hline$x_{21}$ & $(S P, L P)$ & 0 & 0 & 0 & 0 & 0 & 0.5 & 1 \\
\hline$x_{22}$ & $(V S, L N)$ & 0.5 & 1 & 0.5 & 0 & 0 & 0 & 0 \\
\hline$x_{23}$ & $(V S, M N)$ & 0.5 & 1 & 0.5 & 0 & 0 & 0 & 0 \\
\hline$x_{24}$ & $(V S, S N)$ & 0 & 0.5 & 1 & 0.5 & 0 & 0 & 0 \\
\hline$x_{25}$ & $(V S, V S)$ & 0 & 0 & 0.5 & 1 & 0.5 & 0 & 0 \\
\hline$x_{26}$ & $(V S, S P)$ & 0 & 0 & 0 & 0.5 & 1 & 0.5 & 0 \\
\hline$x_{27}$ & $(V S, M P)$ & 0 & 0 & 0 & 0 & 0.5 & 1 & 0.5 \\
\hline$x_{28}$ & $(V S, L P)$ & 0 & 0 & 0 & 0 & 0.5 & 1 & 0.5 \\
\hline$x_{29}$ & $(S N, L N)$ & 1 & 0.5 & 0 & 0 & 0 & 0 & 0 \\
\hline$x_{30}$ & $(S N, M N)$ & 0.5 & 1 & 0.5 & 0 & 0 & 0 & 0 \\
\hline$x_{31}$ & $(S N, S N)$ & 0 & 0.5 & 1 & 0.5 & 0 & 0 & 0 \\
\hline$x_{32}$ & $(S N, V S)$ & 0 & 0.5 & 1 & 0.5 & 0 & 0 & 0 \\
\hline$x_{33}$ & $(S N, S P)$ & 0 & 0 & 0.5 & 1 & 0.5 & 0 & 0 \\
\hline$x_{34}$ & $(S N, M P)$ & 0 & 0 & 0 & 0.5 & 1 & 0.5 & 0 \\
\hline$x_{35}$ & $(S N, L P)$ & 0 & 0 & 0 & 0 & 0.5 & 1 & 0.5 \\
\hline$x_{36}$ & $(M N, L N)$ & 1 & 0.5 & 0 & 0 & 0 & 0 & 0 \\
\hline$x_{37}$ & $(M N, M N)$ & 1 & 0.5 & 0 & 0 & 0 & 0 & 0 \\
\hline$x_{38}$ & $(M N, S N)$ & 0.5 & 1 & 0.5 & 0 & 0 & 0 & 0 \\
\hline$x_{39}$ & $(M N, V S)$ & 0.5 & 1 & 0.5 & 0 & 0 & 0 & 0 \\
\hline$x_{40}$ & $(M N, S P)$ & 0 & 0.5 & 1 & 0.5 & 0 & 0 & 0 \\
\hline$x_{41}$ & $(M N, M P)$ & 0 & 0 & 0.5 & 1 & 0.5 & 0 & 0 \\
\hline$x_{42}$ & $(M N, L P)$ & 0 & 0 & 0 & 0.5 & 1 & 0.5 & 0 \\
\hline$x_{43}$ & $(L N, L N)$ & 1 & 0.5 & 0 & 0 & 0 & 0 & 0 \\
\hline$x_{44}$ & $(L N, M N)$ & 1 & 0.5 & 0 & 0 & 0 & 0 & 0 \\
\hline$x_{45}$ & $(L N, S N)$ & 1 & 0.5 & 0 & 0 & 0 & 0 & 0 \\
\hline$x_{46}$ & $(L N, V S)$ & 1 & 0.5 & 0 & 0 & 0 & 0 & 0 \\
\hline$x_{47}$ & $(L N, S P)$ & 0.5 & 1 & 0.5 & 0 & 0 & 0 & 0 \\
\hline$x_{48}$ & $(L N, M P)$ & 0 & 0.5 & 1 & 0.5 & 0 & 0 & 0 \\
\hline$x_{49}$ & $(L N, L P)$ & 0 & 0 & 0.5 & 1 & 0.5 & 0 & 0 \\
\hline
\end{tabular}

variables, as shown in Table 3 . Note that only the membership functions for nine different values of $\Delta \omega_{u}$ and $\Delta \dot{\omega}_{u}$ are given in Table 3. For a value of $\Delta \omega_{u}$ or $\Delta \dot{\omega}_{u}$ which is not listed in Table 3, linear interpolation must be employed to determine the membership functions.

Let us demonstrate the use of Table 3 by an example. At a particular sampling instant, let the sampled stabili-

Table 3: Membership functions for stabiliser inputs

\begin{tabular}{llllllll}
\hline \multirow{2}{*}{$\begin{array}{l}\text { Normalised inputs } \\
\Delta \omega_{u} \text { or } \Delta \dot{\omega}_{u}\end{array}$} & \multicolumn{6}{c}{ Membership functions } \\
\cline { 2 - 7 } & $L N$ & $M N$ & $S N$ & $V S$ & $S P$ & $M P$ & $L P$ \\
\hline-1.0 & 1 & 0.7 & 0.5 & 0.3 & 0 & 0 & 0 \\
-0.2 & 1 & 0.9 & 0.7 & 0.5 & 0.2 & 0 & 0 \\
-0.1 & 0.8 & 1 & 0.9 & 0.7 & 0.4 & 0.2 & 0 \\
-0.05 & 0.6 & 0.8 & 1 & 0.9 & 0.6 & 0.4 & 0.2 \\
0 & 0.4 & 0.6 & 0.8 & 1 & 0.8 & 0.6 & 0.4 \\
0.05 & 0.2 & 0.4 & 0.6 & 0.9 & 1 & 0.8 & 0.6 \\
0.1 & 0 & 0.2 & 0.4 & 0.7 & 0.9 & 1 & 0.8 \\
0.2 & 0 & 0 & 0.2 & 0.5 & 0.7 & 0.9 & 1 \\
1.0 & 0 & 0 & 0 & 0.3 & 0.5 & 0.7 & 1 \\
\hline
\end{tabular}

IEE PROCEEDINGS, Vol. 137, Pt. C, No. 3, MAY 1990 ser inputs be, say, $\Delta \omega_{u}=0.2$ and $\Delta \dot{\omega}_{u}=-0.1$. From Table 3 , the two stabiliser inputs can be described by the following fuzzy sets.

$$
\begin{aligned}
\Delta \omega_{u}: & \{(L N, 0),(M N, 0),(S N, 0.2),(V S, 0.5), \\
& (S P, 0.7),(M P, 0.9),(L P, 1)\} \\
\Delta \dot{\omega}_{u}: & \{(L N, 0.8),(M N, 1),(S N, 0.9),(V S, 0.7), \\
& (S P, 0.4),(M P, 0.2),(L P, 0)\}
\end{aligned}
$$

\subsection{Determine the membership function of stabiliser} output

Using the previous example, we intend to demonstrate the use of the composition rule to determine the membership function of the stabiliser output. It is obvious from Table 2 that there are 49 rules which can be used to generate the desired stabiliser output. Consider Rule 1 in eqn. 7 or its equivalent, Rule 1 ', in eqn. 8. The 'action' part (the 'Then' part) of the rule has been represented in fuzzy set notations using membership functions but the 'condition' part (the 'If' part) is still to be represented 
using the fuzzy set notation. An observation of Rule 1' reveals that the condition part consists of two predicates ' $\Delta \omega$ is $L P$ ' and ' $\Delta \dot{\omega}$ is $L N$ ' combined together by an 'AND' operator. From eqn. 2 we have the membership value for the condition part.

$$
\begin{aligned}
& \mu\left(x_{1}\right)=\mu\left({ }^{\circ} \Delta \omega \text { is } L P \text { ' and ' } \Delta \dot{\omega} \text { is } L N\right. \text { ') } \\
& =\min \left(\mu\left({ }^{(} \Delta \omega \text { is } L P^{\prime}\right) \quad \mu\left({ }^{\prime} \Delta \dot{\omega} \text { is } L N^{\prime}\right)\right)
\end{aligned}
$$

From eqns. 11 and 12 we have $\mu\left({ }^{\prime} \Delta \omega\right.$ is $\left.L P^{\prime}\right)=1$ and $\mu\left({ }^{\prime} \Delta \dot{\omega}\right.$ is $\left.L N^{\prime}\right)=0.8$. Thus the membership value of the condition part is

$$
\begin{aligned}
\mu\left(x_{1}\right) & =\min (1, \quad 0.8) \\
& =0.8
\end{aligned}
$$

Given the membership values for the 'condition' part and the fuzzy relation matrix, the membership values for the stabiliser output characterised by the seven linguistic variables $L N, M N, S N, V S, S P, M P, L P$ can be obtained using eqn. 5. For example, the membership value for the linguistic variable $L N$ can be computed as follows.

$$
\begin{aligned}
\mu_{V_{\mathrm{PSS}, 1}(L N)} & =\min \left(\mu_{R}\left(x_{1}, L N\right) \quad \mu\left(x_{1}\right)\right) \\
& =\min (0,0.8) \\
& =0
\end{aligned}
$$

Note that this is the membership value of the stabiliser output ' $L N$ ' if only Rule 1 exists. To take the 49 rules in Table 3 into account, the membership values for the "condition' part of all the other 48 rules $\mu\left(x_{i}\right), i=2, \ldots, 49$, must be determined in the same way as we did in eqn. 14 for $\mu\left(x_{1}\right)$. Thus, the final value for stabiliser output ' $L N$ ' can be evaluated using eqn. 5

$$
\mu_{V_{\mathrm{PSS}}}(L N)=\max _{x_{i}}\left(\min \left(\mu_{R}\left(x_{i}, L N\right) \quad \mu\left(x_{i}\right)\right)\right)
$$

The membership values for all the other six variables: $\mu_{V_{\mathrm{PSS}}}(M N), \mu_{V_{\mathrm{PSS}}}(S N), \mu_{V_{\mathrm{PSs}}}(V S), \mu_{V_{\mathrm{PSS}}}(S P), \mu_{V_{\mathrm{PSS}}}(M P)$, and $\mu_{V \text { Pss }}(L P)$ can be computed in exactly the same way. The final results for the preceding example are as follows:

$$
\begin{gathered}
\mu_{V_{\mathrm{PSS}}}(L N)=0.5 \\
\mu_{V_{\mathrm{PSS}}}(M N)=0.7 \\
\mu_{V_{\mathrm{PSS}}}(S N)=0.8 \\
\mu_{V_{\mathrm{PSS}}}(V S)=0.9 \\
\mu_{V_{\mathrm{PSS}}}(S P)=1.0 \\
\mu_{V_{\mathrm{PSS}}}(M P)=0.9 \\
\mu_{V_{\mathrm{PSS}}}(L P)=0.7
\end{gathered}
$$

\subsection{Determine the stabiliser output}

Once the membership values for stabiliser output have been computed, a suitable algorithm must be employed to determine the stabiliser output signal. The algorithm adopted in this work is the 'maximum algorithm' in which the signal with largest membership value is chosen as the stabiliser output signal. Using eqn. 17, the stabiliser output for our example is SP. Since the excitation system can take only numerical values of $V_{\mathrm{PSS}}$, the stabiliser output expressed in linguistic terms must be converted back to numerical values before it can be fed into the excitation system. The conversion table as shown in Table 4 has been compiled based on the stabiliser signals obtained in our previous work on stabiliser design. A different set of numerical values can be selected and different dynamic responses will be obtained. The difference will however be insignificant since the stabiliser signal must be within the narrow range from -0.12 p.u. to $0.12 \mathrm{p}$.u. The table is stored in computer memory as a look-up table. It is observed from Table 4 that the numerical value of stabilising signal for our example is 0.02 p.u.

Table 4: Conversion table from linguistic variables to numerical values

\begin{tabular}{llllllll}
\hline & $L N$ & $M N$ & $S N$ & $V S$ & $S P$ & $M P$ & $L P$ \\
\hline$V_{\text {PSs }}$, p.u. & -0.10 & -0.04 & -0.02 & 0 & 0.02 & 0.04 & 0.10 \\
\hline
\end{tabular}

\section{Example}

Consider a nine-bus, two machine-infinite bus system as shown in Fig. 1 [2]. Fig. 2 depicts the block diagram for the excitation system [2]. The detailed model describing shaft dynamics is not included since the problem of subsynchronous resonance [20-21] is beyond the scope of the present work.

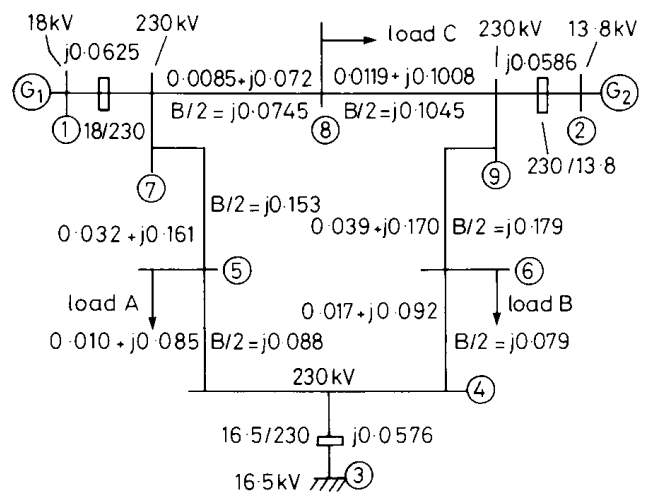

Fig. 1 One line diagram of study system

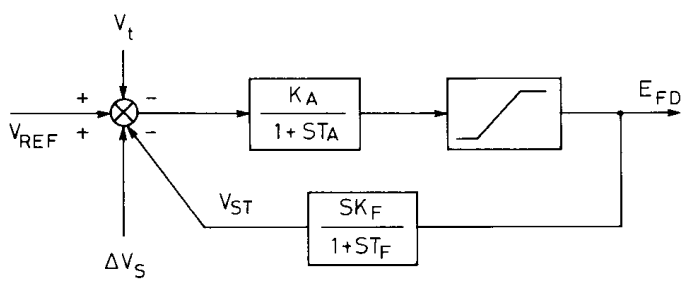

Fig. 2 Static excitation system

The system data are as follows.

Generators

\begin{tabular}{lll} 
generator & 1 & 2 \\
\hline rated MVA & 192.0 & 128.0 \\
$\mathrm{H}(\mathrm{MW} \cdot \mathrm{S} / 100 \mathrm{MVA})$ & 6.4 & 3.01 \\
$\mathrm{KV}$ & 18.0 & 13.8 \\
power factor & 0.85 & 0.85 \\
type & steam & steam \\
speed, rpm & 3600 & 3600 \\
$x_{d}$ & 0.8958 & 1.3125 \\
$x_{d}^{\prime}$ & 0.1198 & 0.1813 \\
$x_{q}^{\prime}$ & 0.8645 & 1.2578 \\
$x_{q}^{\prime}$ & 0.1969 & 0.25 \\
$x_{l}$, leakage & 0.0521 & 0.0742 \\
$\tau_{d o}^{\prime}$ & 6.00 & 5.89 \\
$\tau_{q o}^{\prime}$ & 0.535 & 0.600
\end{tabular}

IEE PROCEEDINGS, Vol. I37, Pt. C, No. 3, MAY 1990 
Excitation systems

$$
\begin{aligned}
K_{A 1} & =K_{A 2}=400 \\
T_{A 1} & =T_{A 2}=0.05 \mathrm{~s} \\
K_{F 1} & =K_{F 2}=0.025 \\
T_{F 1} & =T_{F 2}=1 \mathrm{~s}
\end{aligned}
$$

Loads $(M V A)$

load $A=125+j 50$

load $\mathrm{B}=90+j 30$

load $\mathrm{C}=100+j 35$

The response curves for the system without PSS are shown in Fig. 3. It is observed from Fig. 3, that system damping is poor and it is necessary to install stabilisers in order to have good dynamic performance. In the present study, three different control schemes are investigated for the purpose of comparison.
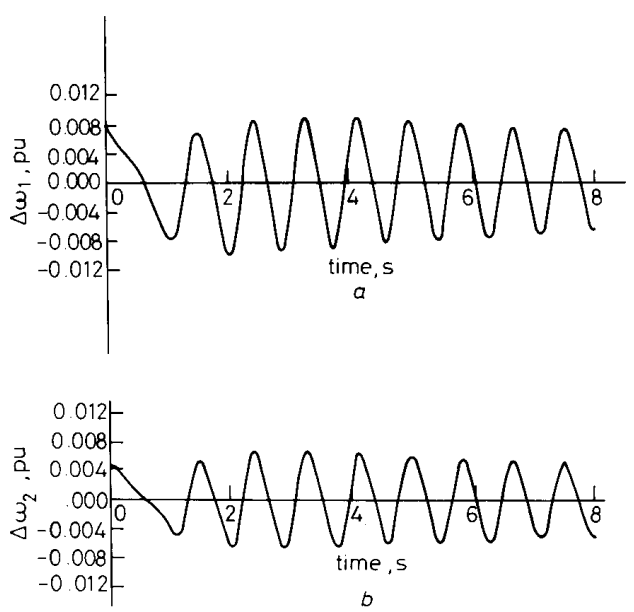

Fig. 3 System response of generators without stabilisers a $\Delta \omega_{1}$

(1) Both generators are equipped with a conventional lead-lag PSS with the transfer function [12]

$$
G(s)=\frac{10 s}{1+10 s} \cdot \frac{(1+0.568 s)^{2}}{(1+0.0227 s)^{2}}
$$
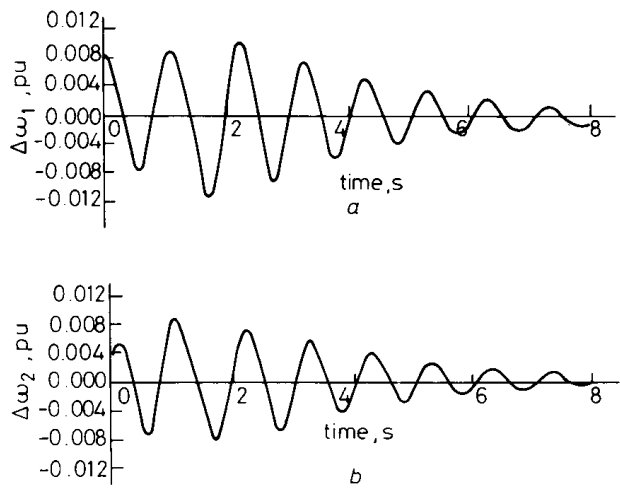

Fig. 4 System response of generators with conventional stabilisers a $\Delta \omega_{1}$

b $\Delta \omega_{2}$

IEE PROCEEDINGS, Vol. 137, Pt. C, No. 3, MAY 1990
(2) Generator 1 is equipped with a fuzzy PSS, while Generator 2 is not equipped with a PSS.

(3) Both generators are equipped with fuzzy stabilisers.

The dynamic responses following a 4 cycle three-phase fault at bus 7 are depicted in Figs. 4-6.
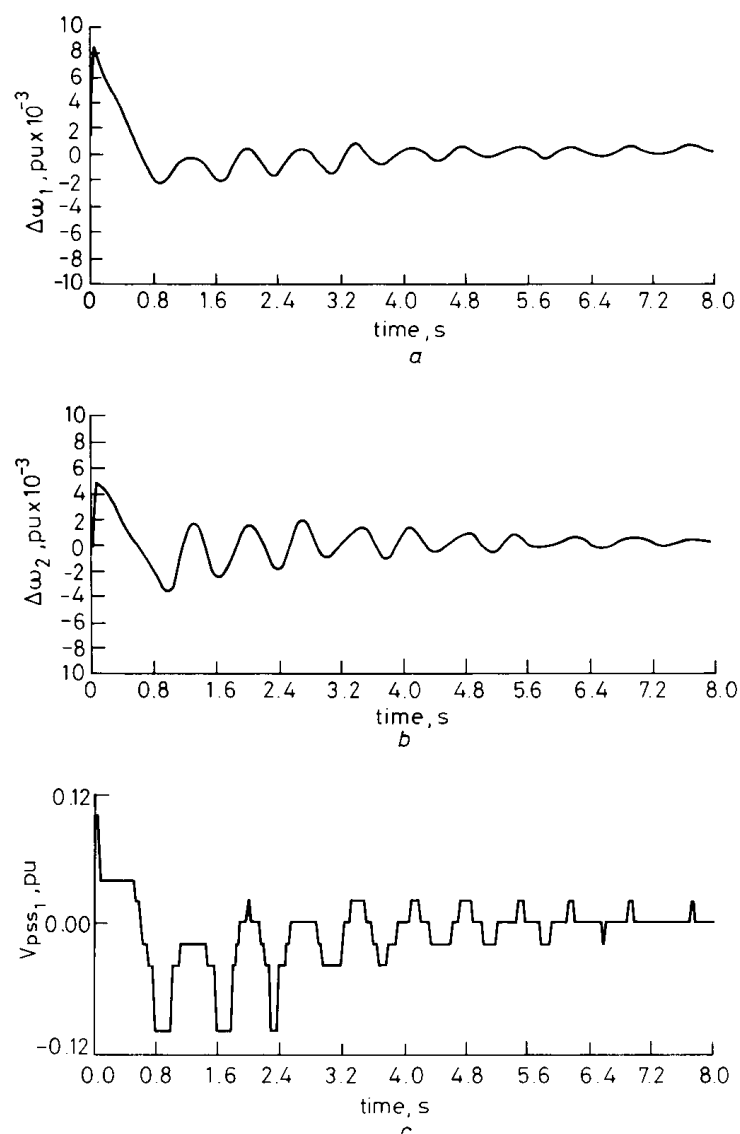

Fig. 5 System response with fuzzy stabilisers on first generator

a $\Delta \omega_{1}$

$\Delta \omega_{2}$

\section{Discussion}

From the response curves in Figs. 3-6, the following observations can be made:

(a) Among the three proposed control schemes, the one with both units equipped with fuzzy stabilisers provides the best damping characteristic.

(b) The dynamic responses of the system with only one generating unit equipped with a fuzzy PSS are still much better than those obtained by using conventional stabilisers.

(c) It can be observed from the response curve of $\Delta \omega_{2}$ in Fig. 5 that, when only generator 1 is equipped with a fuzzy stabiliser, unit 2 will benefit from this fuzzy stabiliser via the transmission links between the two units.

(d) When there is a fuzzy stabiliser on each unit, the two decentralised fuzzy stabilisers co-operate with each other very well in their efforts to damp out system oscillations under disturbance conditions.

(e) As evidenced by the response curves in Figs. 5-6, only moderate gains are required for the fuzzy stabiliser. 
A new approach based on fuzzy set theory has been presented for the design of fuzzy stabilisers which are capable of providing good damping characteristics to a
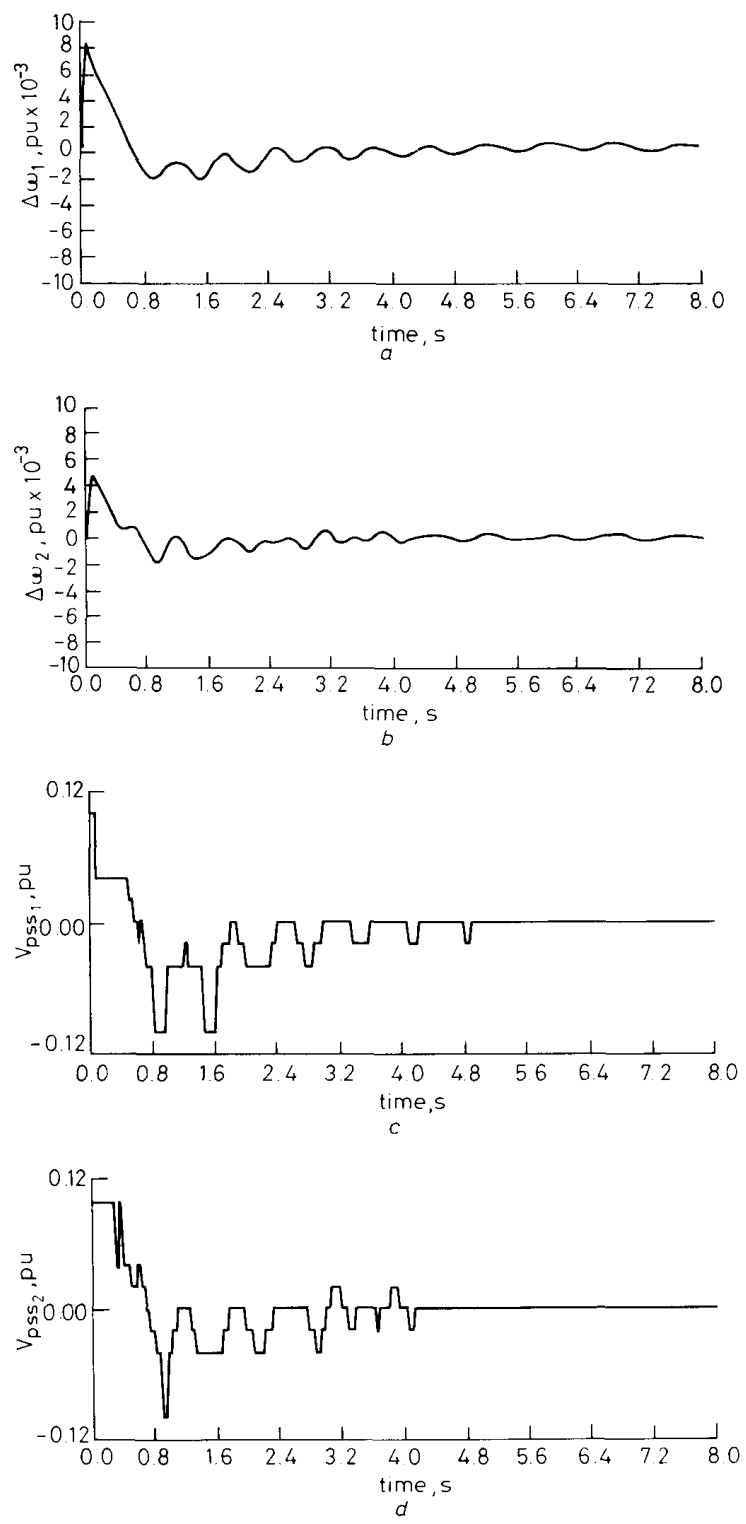

Fig. 6 System response with fuzzy stabilisers on both generators

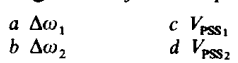

multimachine power system over a wide range of operating conditions. The fuzzy PSS generates its output signal based on some fuzzy decision rules relating stabiliser input signals with stabiliser output. These fuzzy decision rules have been gathered through our previous experience with stabiliser design. Results from this work reveal that, under disturbance conditions, better dynamic performance can be achieved using the proposed fuzzy stabiliser than a conventional stabiliser. The proposed fuzzy stabiliser is very simple for practical implementation since the decentralised output feedback control law developed in this paper requires only local measurements within each generating unit. Moreover, it is superior to a self-tuning stabiliser because it does not require real-time model identification as the self-tuning PSS does. Thus, it is easily implemented on a microcomputer.

\section{References}

1 LARSEN, E.V., and SWANN, D.A.: 'Applying power system stabilisers', IEEE Trans., 1981, PAS-100, pp. 3017-3046

2 ANDERSON, P.M., and FOUAD, A.A.: 'Power system control and stability' (Iowa State University Press, Ames, Iowa, 1977)

3 DEMELLO, F.P., NOLAN, P.J., LASKOWSKI, T.F., and UNDRILL, J.M.: 'Coordinated application of stabilizers in multimachine power systems', IEEE Trans., 1980, PAS-99, pp. 892-901

4 DEMELLO, F.P., and CONCORDIA, C.: 'Concepts of synchronous machine stability as effected by excitation control', IEEE Trans., 1969, PAS-88, pp. 316-329

5 CHAN, W.C., and HSU, Y.Y.: 'An optimal variable structure stabilizer for power system stabilization', IEEE Trans., 1983, PAS-102, pp. $1738-1746$

$6 \mathrm{HSU}$, Y.Y., and HSU, C.Y.: 'Design of a proportional-integral power system stabilizer', IEEE Trans., 1986, PWRS-1, pp. 46-53

7 HSU, Y.Y., SHYUE, S.W., and SU, C.C.: 'Low frequency oscillations in longitudinal power systems: Experience with dynamic stability of Taiwan power system', IEEE Trans., 1987, PWRS-2, pp. 92-100

$8 \mathrm{HSU}$, Y.Y., and SU, C.C.: 'Application of power system stabilizer on a power system with pumped storage plant', IEEE Trans., 1988 , PWRS-3, pp. 80-86

9 XIA, D., and HEYDT, G.T.: 'Self-tuning controller for generator excitation', IEEE Trans., 1983, PAS-102, pp. 1877-1885

10 CHENG, S.J., CHOW, Y.S., MALIK, O.P., and HOPE, G.S.: 'An adaptive synchronous machine stabilizer', IEEE Trans., 1986, PWRS-1, pp. 101-109

11 HSU, Y.Y., and LIOU, K.L.: 'Design of self-tuning PID power system stabilizers for synchronous generators', IEEE Trans., 1987, EC-2, pp. 343-348

12 WU, C.J., and HSU, Y.Y.: 'Design of self-tuning PID power system stabilizer for multimachine power systems', IEEE Trans., 1988 , PWRS-3, pp. 1059-1064

13 HSU, Y.Y., and WU, C.J.: 'Adaptive control of a synchronous machine using the auto-searching method', IEEE Trans., 1988, PWRS-3, pp. 1434-1440

14 CHANDRA, A., MALIK, O.P., and HOPE, G.S.: 'A self-tuning controller for the control of multi-machine power systems', IEEE Trans., 1988, PWRS-3, pp. 1065-1071

15 ZIMMERMANN, H.J.: 'Fuzzy Set Theory and Its Applications' (Kluwer-Nijhoff Publishing Company, 1985)

16 WATSON, W., and MANCHUR, G.: 'Experience with supplementary damping signals for generator static excitation systems', IEEE Trans., 1973, PAS-92, pp. 199-203

17 HIYAMA, T.: 'Rule-based stabiliser for multimachine power system'. Paper 89 SM 691-7 PWRS, presented at the IEEE/PES 1989 Summer Meeting

18 HIYAMA, T.: 'Application of rule-based stabilising controller to electrical power system', IEE Proc. C., 1989, 136, (3), pp. 175-181

19 TANG, K.L., and MULHOLLAND, R.J.: 'Comparing fuzzy logic with classical controller designs', IEEE Trans., 1987, SMC-17, pp. 1085-1087

20 WANG, L., and HSU, Y.Y.: 'Damping of subsynchronous resonance using excitation controllers and static VAR compensators: a comparative study', IEEE Trans., 1988, EC-3, pp. 6-13

21 HSU, Y.Y., and WU, C.J.: 'Design of PID static VAR controllers for the damping of subsynchronous oscillations', IEEE Trans., 1988, EC-3, pp. 210-216 